\title{
Anti-Nociceptive, Analgesic and Pathohistological Effects of Intrathecal Dexmedetomidine and Bupivacaine in Rats
}

\author{
Başar Erdivanli* ${ }^{\text {, }}$ Murat Altun ${ }^{2}$, Özlem K Sezen ${ }^{3}$, Serhan A Çolakoğlu ${ }^{3}$
}

1. MD, Rize Research and Education Hospital, Department of Anesthesiology and Intensive Care

2. MD, Zonguldak Divriği Research and Education Hospital, Department of Anesthesiology and Intensive Care

3. MD, Dr. Lütfi Kırdar Research and Education Hospital, II. Department of Anesthesiology and Intensive Care Received from the Meetings TARK 2009, 28 October - 01 November 2009, Antalya, Turkey.

Submitted on October 29, 2011. Approved on February 27, 2012.

\author{
Keywords: \\ Bupivacaine; \\ Dexmedetomidine; \\ Pain Measurement; \\ Rats, Sprague-Dawley; \\ Anesthesia, Spinal.
}

\begin{abstract}
Background and Objectives: This study investigates analgesic and nociceptive effects of adding dexmedetomidine to bupivacaine neuraxial anesthesia through Tail-flick (TF) and Hot-plate (HP) tests and the pathohistological changes on spinal nerves and nerve roots through light microscopy.

Methods: Forty anesthetized, male Sprague-Dawley rats were intrathecally catheterized. Basal values of TF and HP tests were measured before and after catheterization. Thirty-six successfully catheterized rats were assigned to four groups. Group B received $10 \mu \mathrm{g}$ bupivacaine, Group BD3 received $10 \mu \mathrm{g}$ bupivacaine $+3 \mu \mathrm{g}$ dexmedetomidine, Group BD10 received $10 \mu \mathrm{g}$ bupivacaine $+10 \mu \mathrm{g}$ dexmedetomidine and Control group received $10 \mu \mathrm{L}$ volume of artificial cerebrospinal fluid. TF and HP tests were performed between the $5^{\text {th }}$ and $300^{\text {th }}$ minutes of drug administration. Twenty-four hours after administration of drugs, rats were sacrificed and spinal cord and nerve roots were removed for pathological investigation.

Results: Baseline values of the TF and HP tests were not statistically different among the groups $(6.8 \pm 0.15 \mathrm{~s})$. TF and HP latencies in the Control group did not change significantly during the study. TF and HP test results showed that adding 3 and $10 \mu \mathrm{g}$ dexmedetomidine caused a dosedependent increase in duration and amplitude of analgesic and nociceptive effect of bupivacaine (TF: $37.52 \pm 1.08 \%, 57.86 \pm 1.16 \%$ respectively, HP: $44.24 \pm 1.15 \%, 68.43 \pm 1.24 \%$ respectively) . Conclusions: There were no apparent pathohistological changes at least 24 hours after the intrathecal administration of a single dose of dexmedetomidine $3 \mu \mathrm{g}$ and $10 \mu \mathrm{g}$. Dexmedetomidine added to bupivacaine for spinal block improves analgesia and prolongs block duration.

๑ 2013 Sociedade Brasileira de Anestesiologia. Published by Elsevier Editora Ltda. All rights reserved.
\end{abstract}

*Corresponding author: Rize Eğitim ve Araștırma Hastanesi, İslampasa Mahallesi, Șehitler Caddesi, \#: 74 53100/Rize, Türkiye. Phone: +905057800730, FAX: +904642170364

E-mail: berdivanli@gmail.com

ISSN/\$ - see front metter ๔ 2013 Sociedade Brasileira de Anestesiologia. Published by Elsevier Editora Ltda. All rights reserved. 


\section{Introduction}

Spinal anesthesia is the blockage of nerve transmission through injecting a local anesthetic agent into the cerebrospinal fluid ${ }^{1}$. Its short duration of action and concerns of toxicity resulted in the addition of several adjunct drugs ${ }^{2,3}$. Benzodiazepines ${ }^{4}$, opioids ${ }^{5}$, neostigmine ${ }^{6}$, ketamine ${ }^{7}$ and $\mathrm{a}_{2}$-receptor agonists ${ }^{8,9}$ were used to overcome these shortages. Many of these adjuncts have side effects like itching, urinary retention, respiratory depression, nystagmus, cardiac depression, nausea and vomiting. Lacking many of the above-mentioned side effects and with its safer cerebral and respiratory profile, $a_{2}$-receptor agonists prolong motor and sensory blockade duration, which makes them very popular adjuncts to neuraxial anesthesia.

In this study, we investigated how dexmedetomidine added to bupivacaine neuraxial anesthesia would affect spinal and supraspinal pain pathways through Tail-flick (TF) and Hot-plate (HP) tests and its neurotoxic effect on spinal nerves and nerve roots through light microscopy.

\section{Materials and Methods}

Forty male inbred Sprague Dawley rats (weight, 350-400 g; age, 15-16 weeks) were obtained from Pendik Faculty of Veterinary Science Laboratories (Istanbul, Turkey). The rats were housed in separate cages with unlimited water and food and kept in temperature controlled rooms $\left(20-24^{\circ} \mathrm{C}\right.$, relative humidity $50-60 \%$ ) on a 12 -hour light and 12 -hour dark cycle (dark cycle beginning at 7:00 p.m.). All tests were performed in the morning. The animals were handled according to the "Guide for the Care and Use of Laboratory Animals" prepared by the Institute of Laboratory Animal Resources, National Research Council. The Ethical Committee of Pendik Faculty of Veterinary Science Laboratories, Istanbul, Turkey (Chairperson Dr. Muhammet AKSIN, Ph.D.) gave ethical approval for this study (Ethical Committee No: 13) on 19 January 2009.

The rats were allowed to acclimatize to the laboratory environment for 3 days. Body weights were measured daily throughout the study. On the fourth day, all rats were implanted with an intrathecal (IT) catheter using a modified method of Yaksh ${ }^{10}$. Anesthesia was provided with $60 \mathrm{mg} \cdot \mathrm{kg}^{-1}$ intraperitoneal sodium pentobarbital and periodic supplements of $10 \mathrm{mg} . \mathrm{kg}^{-1}$ of pentobarbital. Response to foot stimulation was monitored to ensure adequacy of anesthesia. In a prone position, the cervical region of rats was shaved and sterilized with povidone-iodine. A longitudinal $2 \mathrm{~cm}$ incision was made and the neck musculature was gently retracted to expose the atlanto-occipital membrane. After we pierced the membrane using a sterile 18-gauge disposable needle, there was an abundant outflow of clear cerebrospinal fluid. A stainless steel wire guide of a spinal needle fitting into the 32-gauge polyurethane (PE32) catheter was used to ease the insertion and prevent the catheter from bending and the traumatization of the spinal cord. The catheter was carefully pushed until vertebral body T11 $(7.4-7.7 \mathrm{~cm})$ into the spinal space from the incision whilst applying slight tension to the tail in order to place the tip at the $T_{11-12}$ vertebral level, estimated to be the $L_{3-4}$ level of the spinal cord. After insertion, we aspirated and flushed it with artificial cerebrospinal fluid (ACSF) prepared using sterile water and adding commercial electrolyte solutions (Merck) to achieve a final concentration of (mEq.L-1) Na: 150, K: 3, Ca: 1.4, Mg: 0.8, P: 1 and Cl: 155 with a $\mathrm{pH}$ of $7.4{ }^{11}$. A bacterial filter was clamped and sealed on the outside end of the catheter. The incision was sutured and the rat returned to a separate cage for 3 days to recover. Rats were checked for motor or sensory deficits after recovery and evaluated daily by using a 4-point scale. Animals with any sign of neurologic deficit, infection, catheter displacement or clogging were discarded. A total of 4 animals were excluded from the study ( 2 because of death during catheterization and 2 because of infection).

Thirty-six rats were randomly assigned into four groups. Randomization procedure was done through computerized equalization of mean body weights of each group of rats. Drugs for IT injection were dissolved in a $10 \mu \mathrm{L}$ volume of ACSF. All drugs were administered over a 1-minute time period. Group B received $10 \mu \mathrm{g}$ bupivacaine, Group BD3 received $10 \mu \mathrm{g}$ bupivacaine $+3 \mu \mathrm{g}$ dexmedetomidine, Group BD10 received $10 \mu \mathrm{g}$ bupivacaine $+10 \mu \mathrm{g}$ dexmedetomidine and Control group received $10 \mu \mathrm{L}$ volume of ACSF on the fourth day after catheterization.

The TF test was structured with a heat source (100 Watt light bulb) focused on the dorsal surface of the tail approximately $4 \mathrm{~cm}$ from the tip. We measured latency in withdrawal from heat source. Lack of occurrence of withdrawal in 14 seconds resulted in termination of the stimulus to avoid damage to the tail.

HP test was structured with a Hot Plate Analgesia Meter from Columbus Instruments (Columbus, $\mathrm{OH}$ ). The surface of the hot plate was heated to a constant temperature of $55^{\circ} \mathrm{C}$. We measured latency to move the hind-paws or jump. The cutoff time was 50 seconds to avoid tissue damage. Baseline values of the TF and HP tests were measured on the first, fourth and seventh days. Post-injection values of the tests at $5^{\text {th }}, 10^{\text {th }}, 15^{\text {th }}, 20^{\text {th }}, 30^{\text {th }}, 45^{\text {th }}$ and $60^{\text {th }}$ minutes and every 30 minutes until the $300^{\text {th }}$ minute were recorded. Results were expressed as percentage of maximum possible effect (\%MPE) according to the following formula: \%MPE = (post-injection latency - pre-injection latency) x 100 / (cutoff time - pre-injection latency). The study was performed double-blindly at the stages of catheterization, drug injection and measurements.

After the tests were completed, animals were put into separate cages and euthanized the next day (approximately 24 hours after injection) with a $1 \mathrm{~mL}$ intra-cardiac dose of $26 \%$ sodium thiopental and the spinal cord removed en bloc via a modified technique as described elsewhere ${ }^{12}$. Briefly, after excising the surrounding skin and muscle tissue, the spinal column was removed and whole lumbar section was separated. The vertebral column was carefully cut. The dorsal and ventral cords were spared and cut from outside the vertebral foramen. During tissue removal, we verified the catheter placement. After tissue fixation in $10 \%$ neutral buffered formalin (Fisher, Pittsburgh, PA) for 48 hours, sections were embedded in paraffin, sliced in 5-10 $\mu$ m thickness, and stained with hematoxylin-eosin, luxol fast blue, trichrome ${ }^{13}$ and Weil's myeline sheath ${ }^{14}$. Longitudinal and parallel sections were obtained at the tip of the catheter, $1 \mathrm{~mm}$ on either side of the tip, and at $2 \mathrm{~mm}$ intervals on either side until a distance of $10 \mathrm{~mm}$ was reached. A neuropathologist blinded to the injections evaluated sections. 


\section{Statistical Analysis}

We used SPSS 17 for Windows to evaluate data. We analyzed starting weights and weight gains of rats throughout the study period using Student's $t$-test. Distributions of TF and HP test results were tested using the Shapiro-Wilk test. Results were analyzed using variance analysis with repeated measures and expressed as mean \pm SEM. We used Tukey's procedure for post hoc comparison. We evaluated motor function, sensorial function and pathohistological results using Fisher's exact test. Differences were considered to be significant at $\mathrm{p}<0.05$.

\section{Results}

We prepared 40 animals and analyzed the results from 36, as four were excluded from the study. Statistical analysis revealed a normal distribution of starting weights, no difference in trends of weight gain and rats' food and water consumption throughout the study.

Evaluations using the 4-point scale showed no sign of neurologic deficit, infection, catheter displacement or clogging. Baseline values of the \%MPE values in the TF and HP tests were not statistically different among the groups (using Student's $t$-test) before drug administration $(4 \pm 1 \mathrm{~s}$ for all groups). TF and HP latencies in the Control group did not change significantly during the study. The measured $\mathrm{pH}$ values were $6.36 \pm 0.05$ for bupivacaine, $6.02 \pm 0.04$ for dexmedetomidine and $6.08 \pm 0.04$ for mixtures of dexmedetomidine and bupivacaine.

During the TF test, $10 \mu \mathrm{g}$ bupivacaine caused a moderate antinociception, which peaked at the $20^{\text {th }}$ min (mean: $48.3 \%$, SEM: $0.9 \%$ ) and was sustained until the $240^{\text {th }} \mathrm{min}$. Addition of $3 \mu \mathrm{g}$ dexmedetomidine to $10 \mu \mathrm{g}$ Bupivacaine caused a high level of antinociception, which peaked at the $30^{\text {th }}$ min (mean: $74.4 \%$, SEM: $1.6 \%$ ) and was sustained until the $300^{\text {th }}$ min. Addition of $10 \mu \mathrm{g}$ dexmedetomidine to $10 \mu \mathrm{g}$ Bupivacaine caused a high level of antinociception, which peaked at the $30^{\text {th }}$ min (mean: $97.9 \%$ SEM: $1.1 \%$ ) and surpassed the $300^{\text {th }}$ min (Figure 1).

A repeated measures ANOVA test with a GreenhouseGeisser correction determined that mean \%MPE levels differed statistically significantly between time points $[F$ $(8.631,45)=12460.737, p<0.0005]$ for the TF test. Post hoc tests using the Tukey's procedure revealed that $10 \mu \mathrm{g}$ bupivacaine caused an increase in \%MPE levels $(22.07 \pm 0.98 \%$ vs. $0.1 \pm 0.1 \%$, respectively) which was statistically significant $(p<0.001)$, addition of $3 \mu \mathrm{g}$ dexmedetomidine to bupivacaine caused an extra increase in \%MPE levels (37.52 $\pm 1.08 \%)$ which was statistically significant $(p<0.001)$, addition of $10 \mu \mathrm{g}$ dexmedetomidine to bupivacaine caused a further increase in \%MPE levels $(57.86 \pm 1.16 \%)$ which was statistically significant $(p<0.001)$.

During HP test, $10 \mu \mathrm{g}$ bupivacaine caused a moderate antinociception, which peaked at the $30^{\text {th }}$ min (mean: $54 \%$, SEM: $1 \%$ ) and was sustained until the $300^{\text {th }}$ min. Addition of $3 \mu \mathrm{g}$ dexmedetomidine to $10 \mu \mathrm{g}$ Bupivacaine caused a high level of antinociception, which peaked at the $30^{\text {th }}$ min (mean: $76.9 \%$, SEM: $1.7 \%$ ) and was sustained until the $300^{\text {th }} \mathrm{min}$. Addition of $10 \mu \mathrm{g}$ dexmedetomidine to $10 \mu \mathrm{g}$ Bupivacaine caused a high level of antinociception, which peaked at the $15^{\text {th }}$ min (mean: $98.7 \%$ SEM: $0.5 \%$ ) and surpassed the $300^{\text {th }}$ min (Figure 2).

A repeated measures ANOVA test with a GreenhouseGeisser correction determined that mean \%MPE levels differed statistically significantly between time points $[F(7.655,45)=12612,953, p<0.0005]$ for the HP test. Post hoc tests using the Tukey's procedure revealed that $10 \mu \mathrm{g}$ bupivacaine caused an increase in \%MPE levels $(28.37 \pm 0.99 \%$ vs. $0.1 \pm 0.1 \%$, respectively) which was statistically significant $(p<0.001)$, addition of $3 \mu \mathrm{g}$ dexmedetomidine to bupivacaine caused an extra increase in \%MPE levels $(44.24 \pm 1.15)$ which was statistically significant $(p<0.001)$, addition of $10 \mu \mathrm{g}$

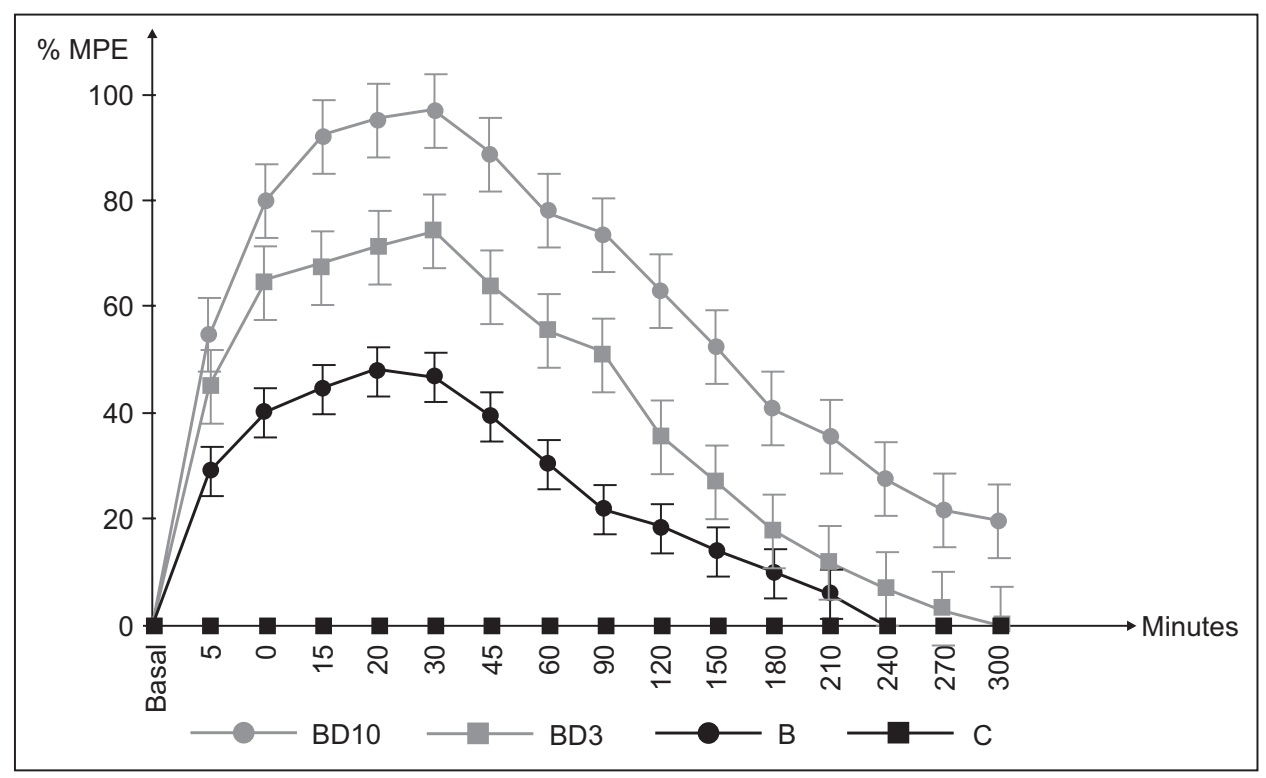

Figure 1 Time course of the antinociceptive effect (\%MPE) of intrathecally administered drugs measured by the TF test. 


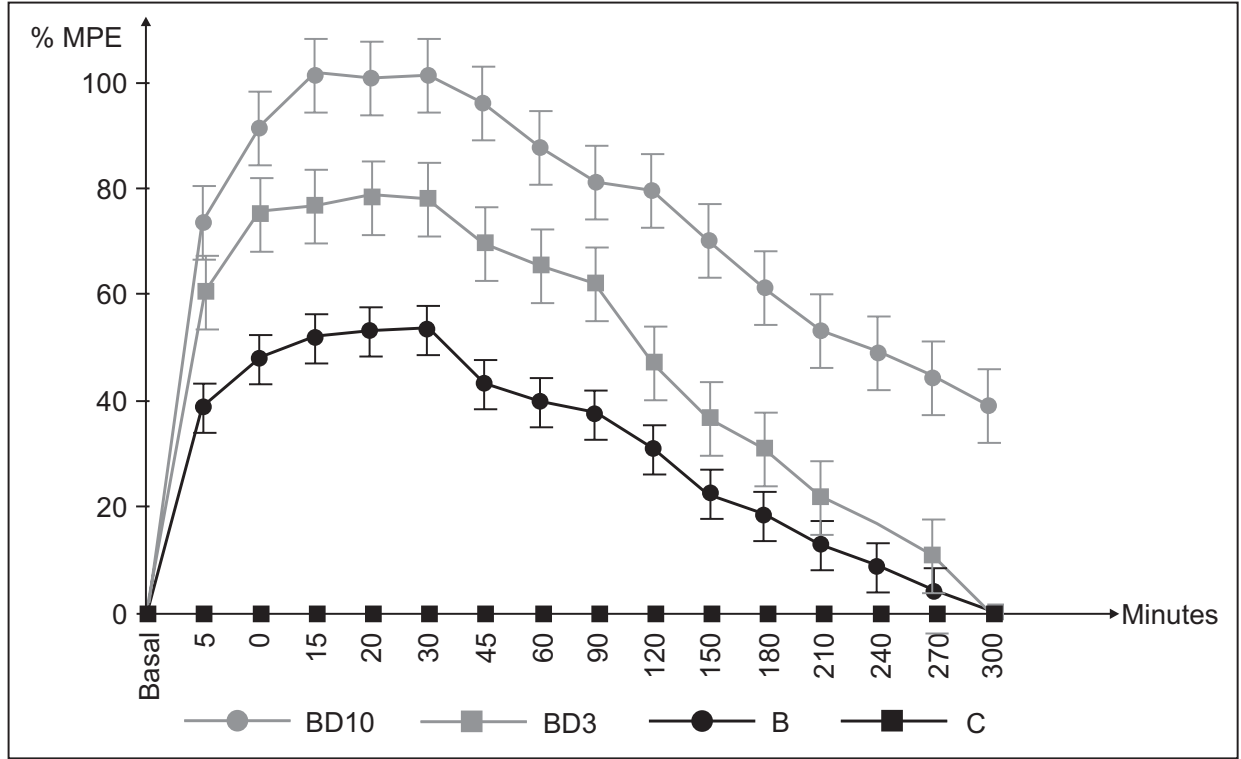

Figure 2 Time course of the antinociceptive effect (\%MPE) of intrathecally administered drugs measured by the HP test.

dexmedetomidine to bupivacaine caused a further increase in \%MPE levels $(68.43 \pm 1.24 \%)$ which was statistically significant $(\mathrm{p}<0.001)$.

At the $24^{\text {th }}$ hour after injections, cellular structure was preserved on all slides. No pathologic change was noted in grey and white matter, dural, pial and arachnoid membranes. Spinal nerve roots and ganglion cells retained their morphology. There was no apparent neurotoxicity at least 24 hours after the administration of single dose of dexmedetomidine $3 \mu \mathrm{g}$ and $10 \mu \mathrm{g}(\mathrm{p}>0.05)$.

\section{Discussion}

This study showed that addition of dexmedetomidine to bupivacaine spinal block enhances the amplitude and duration of nociception to a thermal stimulus. Dexmedetomidine acts on several places to show its antinociceptive action. It binds to presynaptic C-fibers transmitting noxious stimuli from peripheral receptors to spinal cord. It also binds to $a_{2 A}$ - and $a_{2 C}$-adrenoceptors of postsynaptic neurons present in superficial layers of the spinal dorsal horn ${ }^{15}$. Its antinociceptive effects result both from inhibition of presynaptic C-fibers and hyperpolarization of postsynaptic dorsal horn neurons ${ }^{16}$. Ishii and colleagues reported that dexmedetomidine induced activation of $\mathrm{K}^{+}$-channels through $\mathrm{a}_{2 \mathrm{~A}^{-}}$and $\mathrm{a}_{2 c}$-adrenoceptors results in hyperpolarization of substantia gelatinosa neurons ${ }^{15}$. Similarly, Brummett and colleagues reported that dexmedetomidine's antinociceptive action depends on inhibiting the build up of excitation via hyperpolarization-activated cation current $\left(\mathrm{I}_{\mathrm{h}}\right)$ and is reversed by I agonist forskolin ${ }^{17}$.

Among spinal catheterization methods, sacral approach is mainly suited for epidural drug administration and lumbar approach carries the risks of bone damage and inflammation due to fixatives. Therefore the atlanto-occipital approach has been preferred as it was well described by Yaksh et al. ${ }^{10}$. PE32 catheters were used to minimize damage to spinal cord and nerve roots ${ }^{2}$. We chose the spinal route for drug delivery to eliminate the concerns about insufficient diffusion of drugs through dural membrane and also to observe the effect of drugs, $\mathrm{pH}$ and catheters on spinal cord. Several studies verified accurate placement of perineural catheter using lidocaine or any local anesthetic to produce a motor block. In our study, no drug other than the study ones were injected through the catheters to minimize effects, therefore catheter placement was verified by postmortem examination.

Our study showed that dexmedetomidine dose dependently prolonged the duration and augmented the analgesic quality of the spinal block. Our results concord with Calasans and colleagues' study reporting that levobupivacaine-induced spinal block was prolonged by intrathecal dexmedetomidine in doses up to $0.4 \mu \mathrm{g}$ and intraperitoneal doses up to $40 \mu \mathrm{g} . \mathrm{kg}^{-1}$, which was reversed by an a-adrenergic antagonist, yohimbin ${ }^{18}$. These results suggest that dexmedetomidine exerts its effects through $a_{2}$-adrenoceptor agonism. Brummett and colleagues' study investigating addition of dexmedetomidine to ropivacaine induced peripheral nerve block ${ }^{19}$. They administered dexmedetomidine in doses up to $20 \mu \mathrm{g} \cdot \mathrm{kg}^{-1}$, and the $\mathrm{pH}$ value of mixture of ropivacaine and dexmedetomidine was reported to be $5.69 \pm 0.05$, which is far more acidic than the $\mathrm{pH}$ values of drug mixtures measured in our study. This and the fact that a pathohistological examination of nerve tissues were done after $24 \mathrm{~h}$ of drug administration may explain why our study did not show signs of inflammation or nerve damage.

The TF response is organized at the spinal level, the HP response is mediated by both spinal and supraspinal levels ${ }^{20}$. The increase in withdrawal latency times in our study was similar in both TF and HP test results. This may suggest that intrathecally administered dexmedetomidine did not reach the supraspinal centers or that its effects on supraspinal centers were less prominent than on dorsal horn neurons.

In two different studies investigating dexmedetomidine's effects on nerve blocks, onset time of nerve blocks are reported to be shortened by Gupta and colleagues investigating spinal block in lower abdominal surgery and Esmaoglu and 
colleagues investigating axillary nerve blocks ${ }^{16,21}$. In our study, the first measurements in both tests were done at the fifth minute, which prevented the study to show a possible difference between the start times of the anti-nociceptive effect. Since the anesthesia start time is of critical importance in regional anesthesia, frequent measurements starting earlier are needed to clarify any possible difference.

\section{Conclusion}

Dexmedetomidine added to bupivacaine for spinal block improves analgesia and prolongs block duration.

\section{Acknowledgments}

The personnel at Pendik Faculty of Veterinary Science Laboratories have been very helpful in accommodating the animals. Testing equipment for Tail-Flick and Hot-Plate tests were supplied from Marmara University, Medical Faculty, Department of Clinical Pharmacology. The authors declare that there is no conflict of interest.

\section{References}

1. GR Charles BB - Local anesthetics, In: Ronald DM LI, Lee AF, Jeanine PWK, William LY - Miller's anesthesia. Philadelphia, Elsevier, Churchill Livingstone, 2005;573-604.

2. Sakura S - Research on local anesthetic neurotoxicity using intrathecal and epidural rat models. J Anesth, 2007;21(4):533534.

3. Sakura S, Kirihara Y, Muguruma T, et al. - The comparative neurotoxicity of intrathecal lidocaine and bupivacaine in rats. Anesth Analg, 2005;101(2):541.

4. Bharti N, Madan R, Mohanty P, et al. - Intrathecal midazolam added to bupivacaine improves the duration and quality of spinal anaesthesia. Acta Anaesthesiol Scand, 2003;47(9):1101-1105.

5. Karaman S, Kocabas S, Uyar M, et al. - The effects of sufentanil or morphine added to hyperbaric bupivacaine in spinal anaesthesia for caesarean section. Eur J Anaesthesiol, 2006;23(04):285-291.

6. Tan PH, Chia YY, Lo Y, et al. - Intrathecal bupivacaine with morphine or neostigmine for postoperative analgesia after total knee replacement surgery. Can J Anesth, 2001;48(6):551-556.

7. Togal T, Demirbilek S, Koroglu A, et al. - Effects of $s(+)$ ketamine added to bupivacaine for spinal anaesthesia for prostate surgery in elderly patients. Eur J Anaesthesiol, 2004;21(3):193-197.
8. Kanazi G, Aouad M, Jabbour Khoury S, et al. - Effect of low dose dexmedetomidine or clonidine on the characteristics of bupivacaine spinal block. Acta Anaesthesiol Scand, 2006;50(2):222-227.

9. Yoshitomi T, Kohjitani A, Maeda S, et al. - Dexmedetomidine enhances the local anesthetic action of lidocaine via an $-2 a$ adrenoceptor. Anesth Analge, 2008;107(1):96.

10. Yaksh TL,Rudy TA - Chronic catheterization of the spinal subarachnoid space. Physiol Behav, 1976;17(6):1031-1036.

11. Reed D, Withrow C, Woodbury D - Electrolyte and acidbase parameters of rat cerebrospinal fluid. Exp Brain Res, 1967;3(3):212-219.

12. Silva G, Feeney C, Mills L, et al. - A novel and rapid method for culturing pure rat spinal cord astrocytes on untreated glass. J Neurosci Meth, 1998;80(1):75-79.

13. Bancroft JD, Cook HC, Turner DR - Book Manual of histological techniques, Churchill Livingstone Edinburgh, 1984;18-22,4148,225 .

14. Weil A - A rapid method for staining myelin sheaths. Arch Neurol Psychiatr, 1928;20(2):392.

15. Ishii $\mathrm{H}$, Kohno $\mathrm{T}$, Yamakura $\mathrm{T}$, et al. - Action of dexmedetomidine on the substantia gelatinosa neurons of the rat spinal cord. Eur J Neurosci, 2008;27(12):3182-3190.

16. Gupta R, Verma R, Bogra J, et al. - A comparative study of intrathecal dexmedetomidine and fentanyl as adjuvants to bupivacaine. J Anaesthesiol, Clinical Pharmacology, 2011;27(3):339.

17. Brummett $C M$, Hong EK, Janda AM, et al. - Perineural dexmedetomidine added to ropivacaine for sciatic nerve block in rats prolongs the duration of analgesia by blocking the hyperpolarization-activated cation current. Anesthesiology, 2011;115(4):836.

18. Calasans Maia JA, Zapata Sudo G,Sudo RT - Dexmedetomidine prolongs spinal anaesthesia induced by levobupivacaine $0.5 \%$ in guinea pigs. J Pharm Pharmacol, 2005;57(11):1415-1420.

19. Brummett CM, Padda AK, Amodeo FS, et al. - Perineural dexmedetomidine added to ropivacaine causes a dose-dependent increase in the duration of thermal antinociception in sciatic nerve block in rat. Anesthesiology, 2009;111(5):1111.

20. South SM, Edwards SR, Smith MT - Antinociception versus serum concentration relationships following acute administration of intravenous morphine in male and female sprague-dawley rats: Differences between the tail flick and hot plate nociceptive tests. Clin Exp Pharmacol Physiol, 2009;36(1):20-28.

21. Esmaoglu A, Yegenoglu F, Akin A, et al. - Dexmedetomidine added to levobupivacaine prolongs axillary brachial plexus block. Anesth Analg, 2010;111(6):1548. 